\title{
Plan estratégico multisectorial para la reducción de la contaminación acústica por ruido vehicular en la ciudad de Chachapoyas
}

\section{Multisectoral strategic plan for the reduction of noise pollution due to vehicular noise in the city of Chachapoyas}

\author{
Meregildo Silva Ramírez ${ }^{1}$, Rafael Damián Villón Prieto ${ }^{2 *}$ (D) , María Isabel Izquierdo Pacheco ${ }^{2}$ (D)
}

\section{RESUMEN}

El objetivo fue determinar la influencia de un plan estratégico multisectorial (PEM) en la reducción de la contaminación acústica por ruido vehicular en la ciudad de Chachapoyas, sabiendo que la contaminación acústica es un problema que perturba a la sociedad ,que la solución es un trabajo de gestión multisectorial en instituciones, del área educacional con fundamento sociológico, epistemológico, axiológico y antropológico; se ejecutó una investigación aplicada pre experimental; en la población de Chachapoyas, con una muestra probabilística de 229 personas en lugares de confluencia vehicular, que respondieron una encuesta validada de 21 ítems relacionados a efectos físicos, psicológicos y sociales antes y después de la aplicación del PEM, se utilizó un Sonómetro para medir el sonido (dBA) en puntos estratégicos de flujo vehicular registrado a horas $7 \mathrm{am}$ a $8 \mathrm{am}, 12 \mathrm{am}$ a $1 \mathrm{pm}$ y $6 \mathrm{pm}$ a $7 \mathrm{pm}$; registrándose un promedio de $70.52 \mathrm{dBA}$, en la percepción de la sociedad donde existe incomodidad con efectos físicos, psicológicos y sociales, ya que prefieren vivir en el campo que en la ciudad en la evaluación antes $40 \%$ y después 35\%. Se concluye que el PEM tuvo influencia mínima en el control de la contaminación acústica.

Palabras claves: plan estratégico, multisectorial, contaminación acústica, ruido vehicular.

\begin{abstract}
The objective was to determine the influence of a multisectoral strategic plan (PEM) in the reduction of noise pollution by vehicular noise in the city of Chachapoyas, knowing that noise pollution is a problem that disturbs society, that the solution is a job multisectoral management in institutions, in the educational area with a sociological, epistemological, axiological and anthropological foundation; pre-experimental applied research was carried out; In the Chachapoyas population, with a probabilistic sample of 229 people in places of vehicular confluence, who answered a validated survey of 21 items related to physical, psychological and social effects before and after the application of the PEM, a sound level meter was used to measure sound (dBA) at strategic points of vehicular flow recorded at $7 \mathrm{am}$ to $8 \mathrm{am}, 12 \mathrm{am}$ to $1 \mathrm{pm}$ and $6 \mathrm{pm}$ to $7 \mathrm{pm}$; registering an average of $70.52 \mathrm{dBA}$, in the perception of society where there is discomfort with physical, psychological and social effects, since they prefer to live in the country than in the city in the evaluation before $40 \%$ and after $35 \%$. It is concluded that the PEM had minimal influence on the control of noise pollution.
\end{abstract}

Keywords: strategic plan, multisectoral, noise pollution and vehicular

\footnotetext{
${ }^{1}$ Universidad Nacional de Trujillo, Trujillo, Perú

${ }^{2}$ Universidad Cesar Vallejo, Trujillo, Perú

*Autor de correspondencia.E-mail: rafael.villon@gmail.com
} 


\section{INTRODUCCIÓN}

La Organización para la Cooperación y el Desarrollo Económico (OCDE) menciona que 130 millones de personas en un día soportan ruido que supera los 65 decibeles (dBA), nivel establecido por la Organización Mundial de la Salud (OMS) y la Agencia Americana de Protección del Medio Ambiente. Para el año 2011, la OMS reportó que el $40 \%$ de la población de la Unión Europea se expone a niveles superiores a $55 \mathrm{dBA}$ y el $20 \%$ a más de $65 \mathrm{dBA}$, además menciona que aproximadamente el $3 \%$ de casos de enfermedades al corazón se atribuye al ruido vehicular. Específicamente en España más o menos 9 millones de personas soportan un nivel superior a $65 \mathrm{dBA}, 19$ ciudades españolas registran que el $27,7 \%$ de la población soporta ruidos superiores a $65 \mathrm{dBA}$, aproximadamente un tercio de las familias españolas (30,5\%) mencionan permitir incomodidades por ruidos que se dan fuera de las viviendas (Recio y Carmona, 2016). Asimismo, la OMS mencionó que el ruido en exceso pone en riesgo la salud, contribuye a la generación de enfermedades cardiovasculares, por lo tanto, recomienda limitar o reducir la exposición el ruido a $53 \mathrm{dBA}$. En ese sentido lo lamentable es que la contaminación por ruido, casi nunca necesita la atención médica inmediata. Entre 1980 y 1990 alrededor de 4 millones de personas en Estados Unidos perdieron la audición por ruido (We1le, 2018). El ruido es un problema muy evidente que afecta al medio ambiente y es causado por la actividad cotidiana, mediante la maquina en los medios de transporte o la industria, entre otras actividades (Ramírez y Domínguez, 2011). En Europa el ruido es considerado el segundo contaminante más perjudicial (EARPA, 2016). la calidad de vida en un espacio urbano obedece a un proceso de decisiones de autoridades basada en la gestión administrativa de la planificación responsable (Sheina y Fedorovskaya, 2017), siendo necesario tener un registro o mapa detallado del lugar de ocurrencia del ruido para la toma de decisiones preventivas y correctivas (Zamora et al., 2018). En ese sentido se deben resaltar métodos para la disminución del ruido como son los registros de mapeo del ruido y el monitoreo acústico mediante mediciones constantes (Vasilyev, 2017), por su adecuada planificación, la posibilidad de encontrar una ciudad sostenible en relación a la contaminación por ruido vehicular (Barrigón et al., 2018). En casos especiales, como los hospitales, afectan gravemente a los bebés prematuros (Olivera et al., 2011). En Bogotá, se indagó el efecto del nivel de ruido en la atención de 141 estudiantes de una universidad, determinándose que se necesita mejorar las condiciones acústicas del espacio de aprendizaje (Castro et al., 2016), recomendando la simplificación de la información cartográfica y la separación de vehículos ligeros, pesados y motocicletas (Bastián et al., 2016). En la ciudad de Chinchero en Colombia, el ruido vehicular es una problemática porque excede a las normas en un 17\% (Ramírez y Domínguez, 2015). Mientras que en la ciudad de Tarija se determinó que el $39 \%$ de las mediciones esta fuera de los límites permitidos que es 68dBA, llegando a registrar hasta 109 dBA(Román, 2017).

En el Perú, en la ciudad de Tarapoto se realizó la evaluación de la contaminación acústica vehicular en la zona céntrica. Se consideró la zona comercial y de protección especial, se aplicó la técnica de monitoreo tres veces el día (7:00 am - 8:00 am, 12:30 pm - 1:30 pm y 5:00 pm - 6:00 pm) por siete semanas, demostrándose que los resultados están fuera de control según los Estándares de Calidad Ambiental para Ruido, ya que registró $80,4,81,6$ y 87,8 dBA en tres periodos (Delgadillo y Pérez, 2017). A nivel regional y local se efectúo un estudio en la Universidad Nacional Toribio Rodríguez de Mendoza de Amazonas en la ciudad de Chachapoyas. Se identificaron zonas específicas de generación de ruido, lográndose construir un mapa de ruido ambiental (Salas y Barboza, 2014). La ciudad de Chachapoyas tiene una población de 32026 habitantes (INEI, 2017) y un parque automotor de aproximadamente 1200 vehículos (MPCH, 2019) solamente en condición de servicio de taxis. La infraestructura vial urbana se caracteriza en la actualidad por tener calles en mal estado de conservación, lo que genera mayor ruido (Vasilyev, 2017). Asimismo, se define al ruido 
como una mezcla compleja de sonidos con frecuencias variadas (García y Garrido, 2003).

Según el informe de la OMS, considera los $70 \mathrm{dBA}$, como el límite superior aceptable (German y Santillán, 2006). La OMS sostiene que los efectos significativos del ruido en la salud serian los siguiente: Efectos físicos mediante reacciones fisiopatológicas cuando los ruidos producen más de $60 \mathrm{dBA}$, las más frecuentes son mayor velocidad de respiración y pulso, aumento de la presión arterial; Efectos psicológicos como estrés, insomnio, irritabilidad, síntomas depresivos, falta de concentración, siendo los escolares los más afectados. Efectos sociales demostrados en problemas de comunicación y aislamiento (Lechlitner et al., 2016). Por cada $3 \mathrm{dBA}$ por encima de $85 \mathrm{dBA}$, el tiempo de exposición permisible antes de que se produzca un posible daño se reduce por la mitad (Tabla 1) (OHSU, 2019).

Tabla 1. Directrices de tiempo de exposición en decibelios

\begin{tabular}{cc}
\hline $\begin{array}{c}\text { dBA } \\
\text { continuo }\end{array}$ & Tiempo de exposición permitido \\
\hline $85 \mathrm{dBA}$ & 8 horas \\
$88 \mathrm{dBA}$ & 4 horas \\
$91 \mathrm{dBA}$ & 2 horas \\
$94 \mathrm{dBA}$ & 1 hora \\
$97 \mathrm{dBA}$ & 30 minutos \\
$100 \mathrm{dBA}$ & 15 minutos \\
$103 \mathrm{dBA}$ & 7,5 minutos \\
$106 \mathrm{dBA}$ & 3,75 min $(<4$ min $)$ \\
$109 \mathrm{dBA}$ & 1.875 min $(<2 \mathrm{~min})$ \\
$112 \mathrm{dBA}$ & 0.9375 min $(\sim 1$ min $)$ \\
$115 \mathrm{dBA}$ & $0.46875 \mathrm{~min}(\sim 30 \mathrm{segundos})$ \\
\hline
\end{tabular}

En ese sentido la investigación se efectúo para evidenciar o determinar: ¿Cuál es la influencia del plan estratégico multisectorial en la reducción de la contaminación acústica por ruido vehicular en la ciudad de Chachapoyas?; mediante un plan estratégico con acciones basadas en una organización con aliados estratégicos como el Gobierno Regional a través de la dirección de transportes, Municipalidad Provincial, Policía Nacional e Instituciones Educativas y la sociedad en general para lograr convertir el cuidado del medio ambiente relacionado a la contaminación acústica en una forma de vida sostenible; sabiendo por qué que la investigación, se trabajó el cumplimiento del objetivo general que fue: Determinar la influencia del plan estratégico multisectorial en la reducción de la contaminación acústica por ruido vehicular en la ciudad de Chachapoyas, entendiéndose que para su cumplimiento se necesitó de ordenar el trabajo mediante el cumplimiento de objetivos específicos como:(a) Cuantificar el nivel de contaminación acústica por ruido vehicular en la ciudad de Chachapoyas.

(b) Identificar los efectos de la contaminación acústica por ruido vehicular en los ciudadanos de Chachapoyas. (c) Diseñar el plan estratégico multisectorial (PEM). (d)Aplicar el plan estratégico multisectorial para reducir la contaminación acústica por ruido vehicular en la ciudad de Chachapoyas y finalmente. (e) Comparar los resultados del pre y post prueba del plan estratégico multisectorial para reducir la contaminación acústica por ruido vehicular en la ciudad de Chachapoyas.

\section{MATERIALES Y MÉTODOS}

\section{Diseño experimental}

Es un diseño de un solo grupo con una sola medición de la variable dependiente, con prueba de pre y post test.

$$
\mathrm{G} \rightarrow \mathrm{O} 1 \rightarrow \mathrm{X} \rightarrow \mathrm{O} 2
$$

\section{G: Representa la muestra de estudio}

O1: Representa la primera observación (pre test)antes que se aplique el estimulo

$\mathrm{X}$ : Representa el estímulo o sea los efectos del tratamiento

O2: Representa la segunda observación (post test)con aplicación del estímulo.

Técnicas e instrumentos de recolección de datos, validez y confiabilidad

El trabajo se efectúo mediante el tratamiento riguroso de la información, es decir se manejó un proceso que 
asegure la confiabilidad, credibilidad y cientificidad de los momentos de la investigación.

a) Gestión para la adquisición y validación de las herramientas. Estratégicamente en gabinete se diseñó la encuesta que constó de 21 preguntas relacionadas a efectos físicos, psicológicos y sociales de la contaminación acústica.

b) Para el manejo de información y los datos recolectados se procedió a un trabajo responsable de clasificación de los datos mediante la codificación y tabulación de los mismos; el análisis estadístico, la elaboración e interpretación de los datos.

\section{Antes de la aplicación del PEM}

a) Diseñar, validar y aplicar la encuesta, para iniciar la recolección de datos. Con ello, se buscó la forma de evidenciar la existencia de problemas de incomodidad por contaminación acústica por ruido vehicular, problemas asociados al aspecto físico, psicológico y social.

b) Recolección de datos mediante el Sonómetro, con la finalidad de determinar la existencia de contaminación por ruido vehicular en relación a los estándares. Se midió el nivel de intensidad del sonido (Li) y el nivel sonoro continúo equivalente (Leq) durante seis días, en las horas punta, de $7 \mathrm{am}$ a $8 \mathrm{am}$, de $12 \mathrm{am}$ a $1 \mathrm{pm}$ y de $6 \mathrm{pm}$ a $7 \mathrm{pm}$.

c) Procesamiento de datos con la finalidad de proyectar promedios, porcentajes y graficas en relación a los datos de la encuesta y del Sonómetro.

d) Análisis y evaluación de los resultados con la finalidad de encontrar conclusiones para la toma de decisiones en la elaboración o diseño del PEM.

e) Diseño del PEM, en base a los resultados con la finalidad de exponerle a los funcionarios de las instituciones denominadas aliados estratégicos como la Municipalidad, la Policía nacional del Perú, el gobierno regional a través de la dirección regional de Transporte y las Instituciones educativas a nivel secundario. f) Coordinación con los funcionarios de las instituciones aliadas para establecer coordinaciones en bien de la ejecución del PEM.

\section{Después de la aplicación del PEM}

a) Aplicación del PEM y exposición de información en las instituciones aliadas.

b) Aplicación de la encuesta de 21 preguntas

c) Recolección de datos mediante el Sonómetro. Se midió el nivel de intensidad del sonido (Li) y el nivel sonoro continúo equivalente (Leq) durante seis días, en las horas punta.

d) Procesamiento de datos.

e) Análisis y evaluación de los resultados.

f) Diseño final de la propuesta del PEM.

\section{RESULTADOS}

En la figura 1 se observa el registro de decibelios de $\mathrm{Li}$

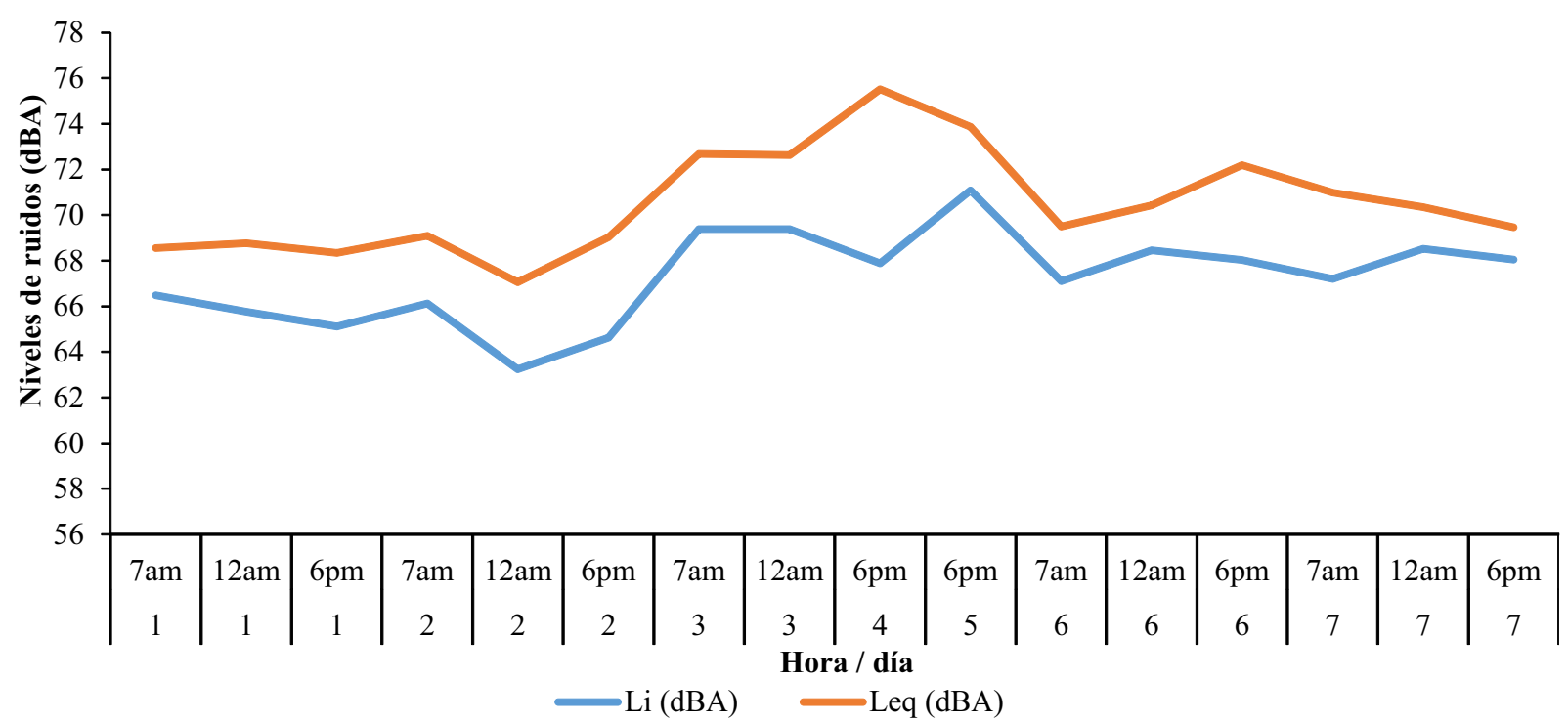

Figura 1. Medición del Li y el Leq del nivel de ruido vehicular (dBA) antes de la aplicación del PEM. 
y Leq, donde el valor máximo del nivel de Leq de ruido vehicular fue de 75,41 dBA. Mientras que el valor máximo del nivel de Li de ruido vehicular fue de 71,08 dBA. Todo esto antes de la aplicación del PEM.

En la figura 2, se muestra el registro de decibelios de $\mathrm{Li}$ y Leq en otro de los cruces más concurridos de la ciduad de Chachapoyas, tras la aplicación del PEM. Se puede observar que el nivel máximo tanto del Li como del Leq fue de 71,37 dBA y 76,60 dBA, respectivamente.

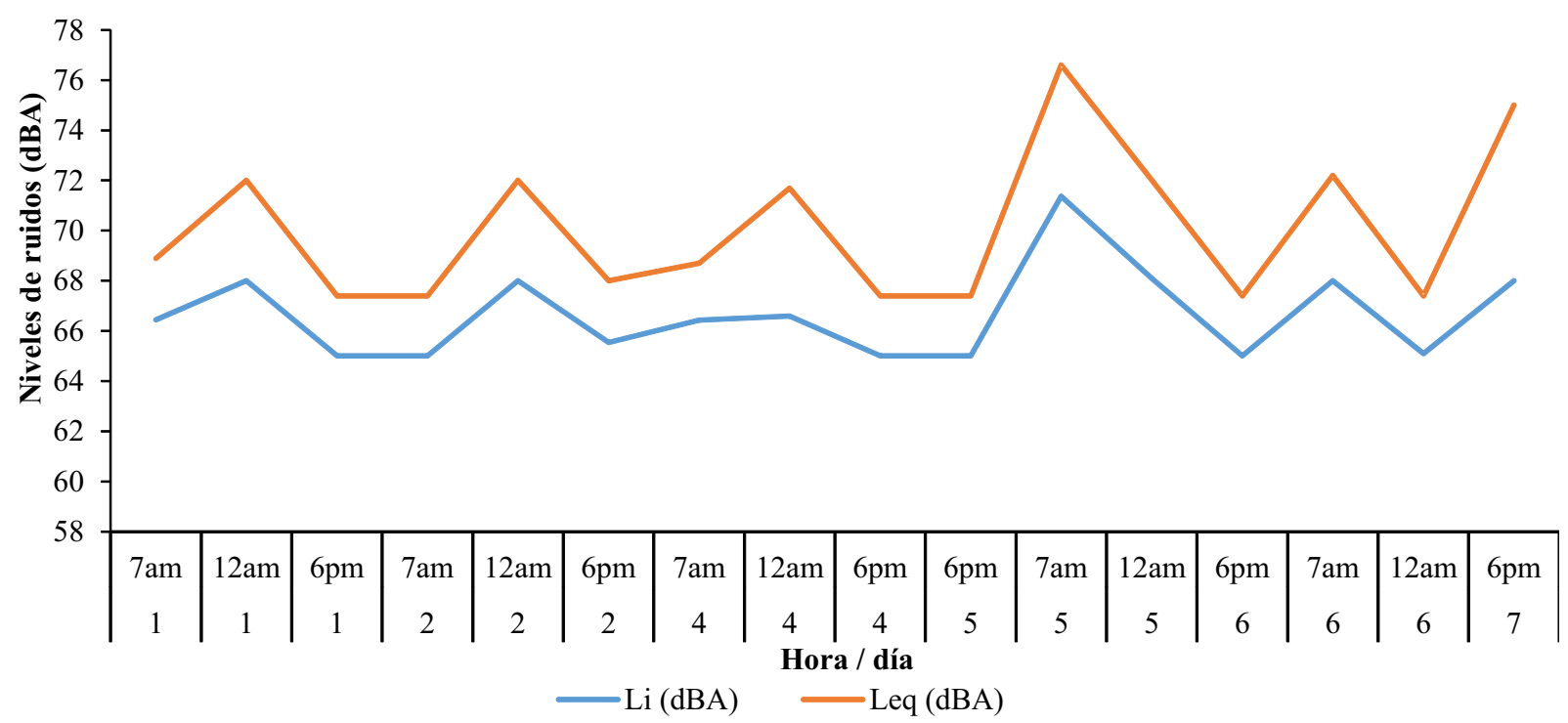

Figura 2. Medición del Li y el Leq del nivel de ruido vehicular (dBA), después de la aplicación del PEM.

En cuanto a los efectos físicos, la mayoría de la población presento alguno de estos efectos ante la presencia del ruido vehicular antes de la aplicación del PEM.

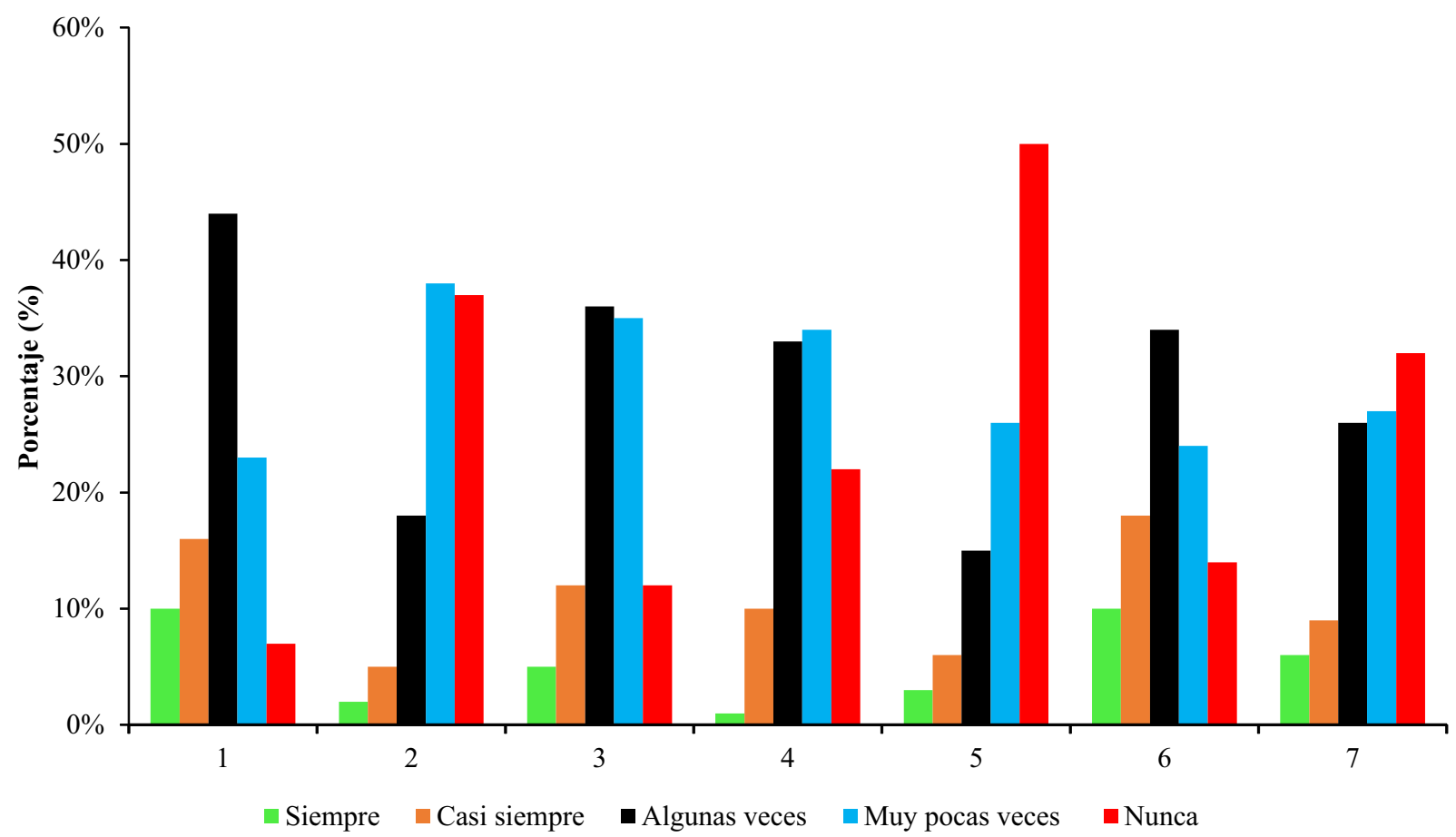

Figura 3. Porcentajes de los efectos físicos de la contaminación acústica por ruido vehicular antes de la aplicación del PEM. (1= Sientes dolores de cabeza a causa del ruido vehicular; $2=$ Tienes dificultad para oír por las noches; $3=$ Tienes dificultades para conciliar el sueño; 4=Notas que ha disminuido tu audición; $5=$ Sientes molestias estomacales ante el ruido vehicular; $6=$ El ruido de los vehículos te causa fatiga; $7=$ Tu respiración se acelera ante el ruido de los vehículos). 
Tras la aplicación del PEM, se observó un descenso de algunos efectos físicos en la población, destacando la dificultad para oír por las noches, donde el $0 \%$ de la población siempre mostro (Figura 4).

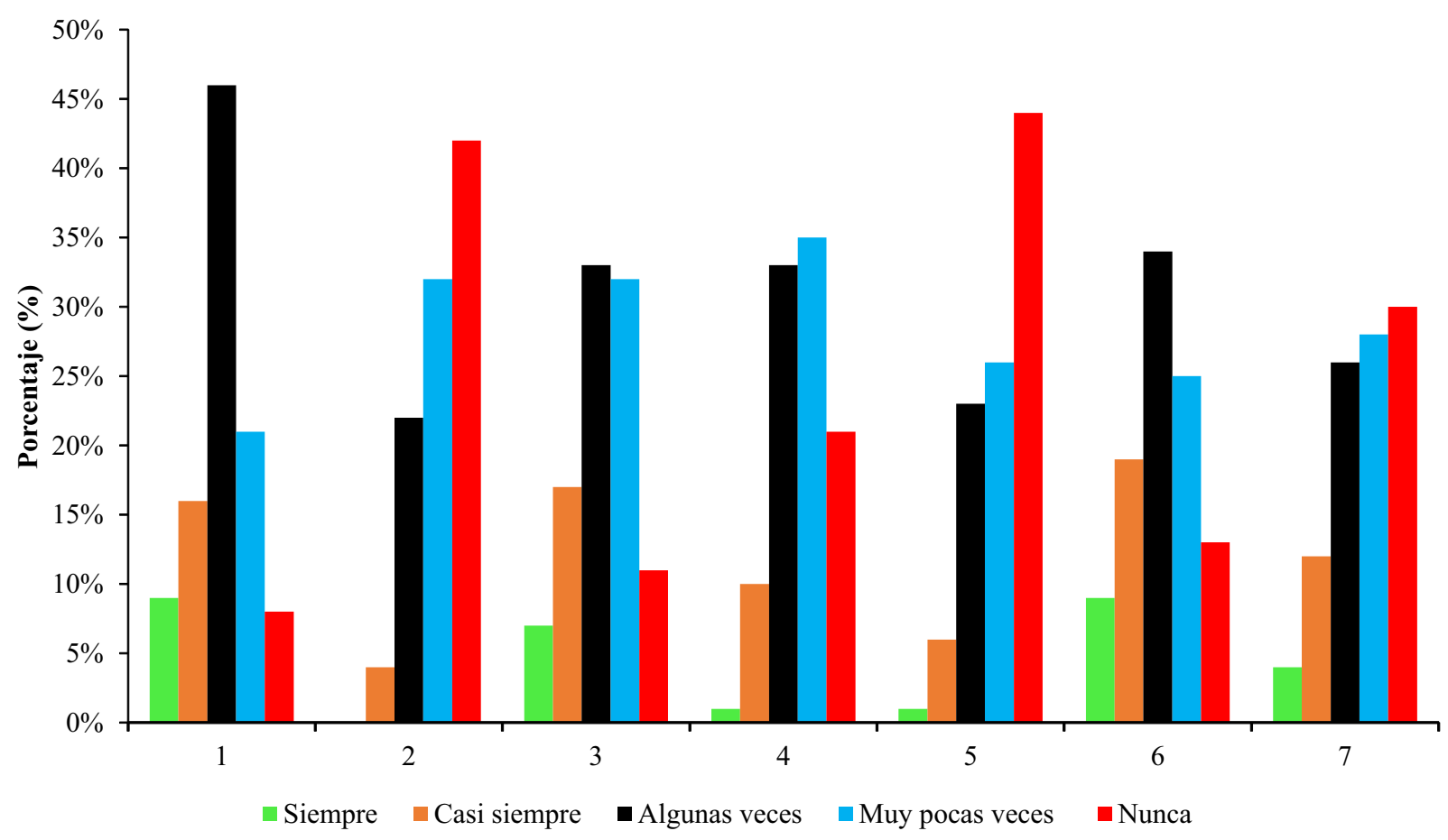

Figura 4. Medición porcentual de los efectos físicos de la contaminación acústica por ruido vehicular después de la aplicación del PEM. (1= Sientes dolores de cabeza a causa del ruido vehicular; $2=$ Tienes dificultad para oír por las noches; $3=$ Tienes dificultades para conciliar el sueño; $4=$ Notas que ha disminuido tu audición; $5=$ Sientes molestias estomacales ante el ruido vehicular; $6=$ El ruido de los vehículos te causa fatiga; $7=$ Tu respiración se acelera ante el ruido de los vehículos).

\section{DISCUSIÓN}

Según los resultados de la investigación se ve que en Chachapoyas existe contaminación ruido vehicular. Sin embargo al ser una ciudad pequeña, aún se puede mencionar que es factible el control y disminución de este problema. Todo ello en base a un Plan estratégico que permita convertir esta dificultad, en una oportunidad para desarrollar la ciudad de forma desarrollo sustentable. mediante una cultura de la prevención y del cuidado del medio ambiente y por ende la salud de las personas.

Según Aramayo (2017), un plan estratégico debe presentar características en la planeación, como son la visión de futuro, una filosofía como cultura organizacional, un manejo metodológico con etapas y resultados, y la flexibilidad para el cambio al momento necesario. De acuerdo a los resultados de la ciudad de Chachapoyas, se propuso un PEM en el contexto actual de la ciudad, teniendo en cuenta que se determinó estándares de ruido vehicular obteniendo un nivel promedio de 75,41 dBA a 76,60 dBA. Sin embargo, para que efectivamente tenga resultados considerables se necesita de un trabajo efectivo. Principalmente con el logro de compromisos e identificación con lo propuesto por parte de los involucrados, se necesita que se implemente como se menciona una cultura de organización, para favorecer el desarrollo sustentable.

Según Pastor (2005), en la ciudad de Trujillo en Perú, las personas están expuestas a niveles de ruido entre 79,8 y 85,4 dBA. Si se realiza una comparación con la ciudad de Chachapoyas, lamentablemente podría calificarse como una situación alarmante, ya que los niveles de ruido vehicular medidos, son muy similares a pesar de ser una ciudad pequeña en comparación a la ciudad de Trujillo. Por su puesto, esta realidad se evidencia en los resultados de incomodidad que sienten las personas frente al ruido vehicular en Chachapoyas. Se puede observar que el Leq antes de la aplicación de PEM es de 75,41 dBA, mientras que después de la aplicación del PEM, el Leq fue mayor con un valor de 76,60 dBA, esto se sustenta al incremento de la población rural hacia lo urbana por ser una ciudad cosmopo- 


\section{lita (MPCH, 2019).}

Teniendo como fundamentos o enfoques teóricos aplicados al PEM, enfoques sociológicos, epistemológicos, axiológicos y antropológicos. Como ejes fundamentales y como principios se tiene la visión de futuro, la visión integral, la filosofía de acción, la efectividad y la flexibilidad. Y como ejes estratégicos, a las políticas locales y acciones compartidas y a la vez sostenidas por los pilares de complementariedad institucional, cultura ambiental y política pública siendo sostenido por los aliados estratégicos como las municipalidades, los gobiernos regionales, las instituciones educativas, y la policía nacional, para unir esfuerzos en reducir la contaminación acústica vehicular en una ciudad, como puede ser la de Chachapoyas. En comparación con Weber (1999) y Giner (2004), los fundamentos o enfoques teóricos, visto en los seres humanos es un proceso lento tanto en lo sociológico, epistemológico, axiológico y antropológico y en las encuestas aplicadas a las 229 personas los resultados muestran que todo pasa por enfoques teóricos, y los países en desarrollo es una cultura ambiental de proceso formativo y estamos todos comprometidos en el cuidado del medio ambiente.

Santos (2007), en la ciudad de Lima determinó que el ruido genera molestias en la población urbana, para ello se realizó encuestas a los transeúntes y conductores en horas punta, recomendándose además que se necesita de voluntad para solucionar este problema complejo. En Chachapoyas, los resultados son similares a la ciudad de Lima, donde las personas expresan su molestia, sin embargo la comparación sería un caso especial porque la densidad del parque automotor en bastante mayor en la ciudad de Lima y con razón las personas expresan su molestia. Sin embargo existe la contaminación acústica a pesar de ser una ciudad pequeña. La probable explicación, se debería a la falta de educación vial y ambiental. En un espacio más complejo de mencionar podríamos atribuirle a la cultura desordenada de los conductores de vehículos o también a la impertinencia de los transeúntes.

\section{CONCLUSIONES}

En el estudio de campo con el sonómetro realizado en las calles de mayor concurrencia vehicular de taxis se obtuvo el nivel de ruido vehicular promedio en 70.52 dBA antes de la aplicación del PEM y después de la aplicación del PEM se obtuvo el nivel acústico de 69.91 dBA.

Se realizó una encuesta a 229 personas de 21 preguntas con efectos físicos, psicológicos y sociales referentes al ruido vehicular donde se obtuvo resultados del antes y después con variaciones porcentuales en dichos efectos, donde las personas prefieren vivir en el campo que en la ciudad; aquí se evidencio un declive de $40 \%$ a $35 \%$, esto indica de que el PEM de alguna manera influye en los efectos sociales de la población.

Según la OMS los niveles deben ser como máximo 60 Dba a $65 \mathrm{dBA}$ y el estudio realizado en campo en la ciudad de Chachapoyas nos cuantifica un Leq promedio de 70.52 dBAa 69.91 dBA.

Se diseñó el PEM que tiene como principios la visión de futuro, visión integral, filosofía de acción, efectividad y flexibilidad, dos ejes estratégicos, como las políticas locales y acciones compartidas. Se articulan cinco fases: primero el diagnóstico, segunda fase la formulación, tercera fase formalización, como penúltima fase ejecución y quinta fase evaluación que es el momento donde se hace de conocimiento el valor promedio de $69.91 \mathrm{dBA}$ del ruido en la ciudad de Chachapoyas, el proceso de monitoreo y los resultados de evaluación de salida (post test). Cumplido este plan PEM se habría prevenido la contaminación acústica por ruido vehicular de manera a controlar los efectos físicos, psicológicos y sociales.

\section{REFERENCIAS BIBLIOGRÁFICAS}

Aramayo, A. 2017. Planeamiento estratégico empresarial. Lima (Perú): Veritas

Barrigón, J. M., G. G. Rey, D. Montes, P. Atanasio, y R. Vílchez. 2018. "Noise Pollution and Urban Planning”. Current Pollution Reports 4 (3): 208-219.

Bastián, N. A., J. Arenas, y E. Suárez. 2016. 
"Assessment of methods for simplified traffic noise mapping of small cities: Casework of the city of Valdivia, Chile". Science of The Total Environment 550: 439-448. DOI: 10.1016/j.scitotenv.2016.01.139

Castro, J. A., J. Chavarría, A. Parra, y S. González. 2016. "Effects of classroom -acoustic change on the attention level of university students". Interdisciplinaria: 33 (2): 201-214.

Delgadillo, M. C., y A. E. Pérez. 2017. “Evaluación de contaminación sonora vehicular en el centro de la ciudad de Tarapoto, San Martín, 2015”. Revista de Investigación Ciencia, Tecnología y Desarrollo 3 (2): 33-47

García, B., y F. J. Garrido. 2003. La contaminación acústica de nuestras ciudades. Barcelona (España): Fundación La Caixa.

German, G., M., y A. Santillán. 2006. “Del concepto de ruido urbano al de paisaje sonoro". Revista Bitácora Urbano Territorial 1 (10): 39-52.

Giner, S. 2004. "El logro de la sociología." INTERthesis 1 (1): 1-36.

INEI (Instituto Nacional de Estadística e Informática). 2017. Censo de población y vivienda. Lima (Perú): INEI

EARPA (European Automotive Research Partners Association) 2016. Noise, vibration and harshness research needs, priorities \& challenges. Informe Técnico. Bruselas (Bélgica).

Lechlitner, S., M. McCullagh, V. Vaughan, y J. Xu. 2016. "Position statement: Harmful effects of environmental noise exposures." American Academy of Nursing on Policy. 64 (4): 395396.

MPCH (Municipalidad Provincial de Chachapoyas). 2019. Plan Operativo Institucional. Informe. Chachapoyas (Perú)

OH S . 2019 . Virtual Exhibit. http://www.dangerousdecibels.org/virtualex hibit/ (Consultada el 12 de diciembre de 2019)

Olivera, J. M., L. A. Rocha, V. I. Rotger, y M. C.
Herrera. 2011. "Acoustic pollution in hospital environment". Journal of Physics: Conference Series. 332 (1): 1-10.

Pastor, J. A. 2005. Efectos de la contaminacion acústica sobre la capacidad auditiva de los pobladores de la ciudad de Trujillo. Tesis de Doctorado. Universidad Nacional de Trujillo. Trujillo (Perú)

Ramírez, A., y E. A. Domínguez. 2015. “Contaminación acústica de origen vehicular en la localidad de Chapinero (Bogotá, Colombia)." Gestión y Ambiente 18 (1): 17-28.

Ramírez, A., y E. A. Domínguez. 2011. “El ruido vehicular urbano: problemática agobiante de los países en vías de desarrollo". Revista de la Academia Colombiana de Ciencias Exactas, Físicas y Naturales 35 (137): 509-530.

Recio, A., y R. Carmona. 2016. "Efectos del ruido urbano sobre la salud: Estudios de análisis de series temporales realizados." Informe de Investigación. Madrid (España).

Román, G. 2017. "Evaluación de los niveles de ruido ambiental en casco urbano de la ciudad de Tarija, Bolivia". Acta Nova 8 (3): 421-432.

Salas, R., y E. Barboza. 2014. "Evaluación del ruido ambiental en el Campus de la Universidad Nacional Toribio Rodríguez de Mendoza de Amazonas, Perú.” Revista INDES 2 (1): 8896.

Santos, E. 2007. “Contaminación sonora por ruido vehicular en la Avenida Javier Prado". Diseño y tecnología 10 (1): 11-15.

Sheina, S., y A. Fedorovskaya. 2017. "Ecological aspects in assessment of acoustic pollution at the territory of Rostov-on-Don." MATEC Web of Conferences 106: 07010. DOI: $10.1051 /$ matecconf $/ 201710607010$

Vasilyev, A. 2017. "New Methods and Approaches to Acoustic Monitoring and Noise Mapping of Urban Territories and Experience of it Approbation in Conditions of Samara Region 
of Russia.” Procedia Engineering 176: 669-

674. DOI: 10.1016/j.proeng.2017.02.311

Weber, M. 1999. The methodological foundations of sociology. Nueva York(EE.UU.): Continuum

Welle, D. 2018. La OMS recomienda limitar exposición a ruidos excesivos por su impacto en la salud. https://prodavinci.com/la-omsrecomienda-limitar-exposicion-a-ruidosexcesivos-por-su-impacto-en-la-salud (Consultada el 10 de octubre de 2018)

Zamora, W., E. Vera, C. T. Calafate, J. C. Cano, y P. Manzoni. 2018. "GRC-Sensing: An Architecture to Measure Acoustic Pollution Based on Crowdsensing." Sensors 18 (8): 2596. DOI: $10.3390 / \mathrm{s} 18082596$ 